
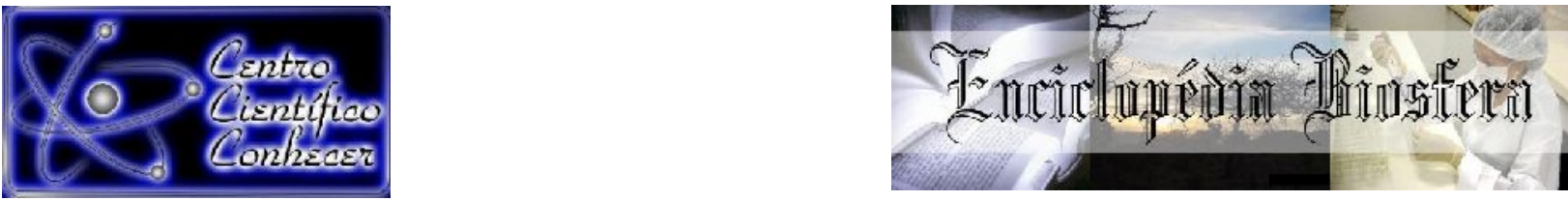

\title{
LINGUAGEM BRASILEIRA DE SINAIS PARA ATENDIMENTOS DE URGÊNCIA E EMERGÊNCIA
}

Karina Rodrigues dos Santos ${ }^{1}$, Severino Cavalcante de Sousa Júnior ${ }^{2^{*}}$, Carlos Syllas Monteiro Luz ${ }^{4}$, Marcelo Richelly Alves de Oliveira ${ }^{5}$, Maria Patrícia Freitas de Lemos ${ }^{3}$

${ }^{1}$ Professora Doutora do curso de Medicina da Universidade Federal do Piauí/Campus Ministro Reis Velloso, Parnaíba, Brasil: krsantos2004@yahoo.com.br.

${ }^{2}$ Professor Doutor do curso de Medicina da Universidade Federal do Piauí/Campus Ministro Reis Velloso, Parnaíba, Brasil.

${ }^{3}$ Professora Doutora do curso de Pedagogia da Universidade Federal do

Piauí/Campus Ministro Reis Velloso, Parnaíba, Brasil

${ }^{4}$ Doutorando do programa de pós graduação em produção animal/CCA, Teresina, Brasil

${ }^{5}$ Doutorando do programa de pós graduação em produção animal/CCA, Teresina, Brasil

\section{Recebido em: 06/04/2019 - Aprovado em: 10/06/2019 - Publicado em: 30/06/2019 DOI: 10.18677/EnciBio_2019A159}

\begin{abstract}
A linguagem brasileira dos sinais (Libras) é a segunda língua oficial do Brasil, e fundamental para o processo de inclusão e socialização dos portadores de deficiência auditiva, que em 2010 representavam mais de $5 \%$ da população brasileira. Foram aplicados questionários aos participantes, da área da saúde, do I workshop de LIBRAS para o atendimento de urgência e emergência, que foi realizado no período de 26 a 28 de setembro de 2015. Os questionários foram respondidos, antes e depois das palestras, e foi utilizada a metodologia qualitativa para análises dos resultados e aplicado o teste de qui-quadrado. Foram encontradas diferenças significativas nos resultados em dois momentos diferentes, antes e depois da apresentação das palestras, significando que o mecanismo de transmissão de conhecimentos e divulgação de informações foi eficiente para os profissionais da área da saúde presentes no Workshop.
\end{abstract}

PALAVRAS-CHAVE: emergência, libras, saúde, urgência.

\section{BRAZILIAN LANGUAGE SIGN FOR EMERGENCY CARE AND EMERGENCY}

\section{ABSTRACT}

The Brazilian language of signs (Libras) is the second official language in Brazil, and fundamental to the inclusion and socialization process of hearing impaired individuals, who in 2010 represented more than $5 \%$ of the Brazilian population. Questionnaires were applied to the participants, in the health area, of the I LIBRAS 
workshop for the emergency and urgency care, which was held at in the period from September 26th to 28th, the questionnaires were answered, before and after the lectures, and qualitative methodology was used for analysis of the results and the Chi-square test was applied. Significant differences in two different moments, before and after the lectures presentation, Meaning that this mechanism of knowledge transmission and dissemination of information was efficient for health care workers present at the Workshop.

KEYWORDS: emergency, libras. health. urgency.

\section{INTRODUÇ̃̃O}

De acordo com a Organização das Nações Unidas ONU (2017), o número estimado de pessoas com alguma deficiência no mundo é de aproximadamente 600 milhões, e $80 \%$ desta parcela populacional vive em países em desenvolvimento, como o Brasil (BERNARDES et al., 2009). O meio de comunicação mais comumente utilizado entre pessoas é a língua falada, esta permite a troca de informações entre as pessoas. A diferença entre língua falada e a linguagem de sinais, é que esta faz uso da visão como fonte receptora e não a audição como acontece na linguagem oral. Os deficientes auditivos utilizam como linguagem padrão a língua de sinais, chamada de LIBRAS (Linguagem Brasileira de Sinais), língua que permite a expressão de quaisquer conceitos, seja descritivo, emotivo, racional, literal, metafórico, concreto ou abstrato (CRUZ et al., 2016).

A deficiência auditiva acarreta em graves problemas sensoriais que resultam em dificuldades de comunicação através da linguagem oral normal, gerando a necessidade de comunicação por sinais, ou LIBRAS (MOREIRA, 2017). A linguagem padrão utilizada pelos surdos torna este grupo prejudicado, dificultando assim o seu acesso a locais como hospitais, dificultando a comunicação do surdo com o profissional de saúde durante o atendimento tanto ambulatorial quanto em um atendimento de urgência, onde os profissionais de saúde normalmente não possuem entendimento aceitável da linguagem de sinais (OLIVEIRA_et al., 2015).

O contato entre o paciente surdo e o profissional de saúde geralmente ocorre de uma forma inadequada pelo fato dos profissionais desconheceram a LIBRAS, e esses profissionais se percebem limitados dificultando o vínculo entre ele e o paciente. Diante desta dificuldade muitos profissionais necessitam de auxilio e para tanto muitas vezes a única solução é incluir uma terceira pessoa na relação paciente e profissional da saúde que seria solicitar auxilio de um interprete para intermediar a consulta (CHAVEIRO et al., 2010).

Um grande problema é que esse profissional no Brasil, na maioria das vezes não possui formação adequada para atuar na área de saúde. Sem considerar que muitas vezes o paciente se sente constrangido com esta terceira pessoa, pois pode querer manter sigilo, por exemplo, em uma consulta com um psicólogo, ou em uma consulta mais intima como com uma ginecologista (CHAVEIRO et al., 2010). Além do que muitos desses profissionais são apenas voluntários e não recebem nada para fazerem o papel de interpretes e quando são remunerados na maioria das vezes é pela família do paciente, porque os surdos não recebem serviços gratuitos dos estabelecimentos (QUEIROZ, 2014).

Nos Estados Unidos existe a presença de interpretes nos hospitais tornando a acessibilidade mais clara e, além disso, a administração pública reembolsa os pacientes. Existem diversos programas de treinamento para interpretes médicos, e 
estes são realidades um pouco distantes, porém se não fosse, poderiam assegurar ao paciente uma consulta e um atendimento com mais qualidade (QUEIROZ, 2014).

E em se tratando de Brasil a Língua Brasileira de Sinais ou simplesmente LIBRAS, como é conhecida, é hoje uma barreira para os profissionais da área da saúde, no que diz respeito ao atendimento de urgência e emergência, tanto nos hospitais públicos e privados como nas unidades básicas de saúde (UBS), pois estas não apresentam equipes especializadas e treinadas pera este atendimento diferencial. O que torna evidente a falta de preparo destes profissionais para atendimentos de pacientes com deficiência auditiva (SILVA; FARIA, 2014).

A necessidade da comunicação, na relação médico paciente, é extremamente importante para o sucesso dos atendimentos, sejam estes, primeiros socorros ou atendimentos mais rotineiros. A transmissão de informações, entre médico paciente, é a ferramenta mais antiga utilizada pela medicina, uma vez que esta arte teve início em épocas que ainda não existiam exames de imagem, som e outros. A medicina sempre utilizou a comunicação com os pacientes como o principal mecanismo de busca de enfermidades, com o objetivo de identificar possíveis patogenicidades e identificar doenças de maneira mais direta e eficiente, o nome dado a este mecanismo é anamnese (CRUZ et al., 2016).

Os surdos e os deficientes auditivos de Parnaíba vivem uma realidade histórica de nível baixíssimo de escolaridade, além da escassez de profissionais na área de educação, faltam nas escolas regulares de ensino fundamental e médio, professores e intérpretes de LIBRAS (Língua Brasileira de Sinais) (APAS, 2017). Com a aprovação da lei municipal de Parnaíba, no 2.649/11, a APAS - Associação de Pais e Amigos dos Surdos, atuante na defesa dos direitos das pessoas surdas e na promoção da sua acessibilidade, desenvolve o curso de capacitação de docentes para Comunicação com alunos surdos, um curso presencial especialmente elaborado para os docentes e técnicos (APAS, 2017).

Os serviços de saúde e as equipes de profissionais da saúde devem estar preparados para atuarem de forma a garantir um atendimento digno e qualidade da saúde dos deficientes, por meio do conhecimento do segundo idioma oficial da República Federativa do Brasil, que é a Linguagem dos Sinais do Brasil (LIBRAS), este conhecimento se faz necessário e importante para os profissionais da área da saúde e áreas afins, pois, além da participação direta na melhoria da qualidade de vida destes pacientes, os médicos, enfermeiros, fisioterapeutas, biomédicos, psicólogos e demais profissionais devem ainda entender os sinais dos pacientes para que possam atuar, de maneira correta e eficaz, para com este público especial e cada vez mais normal (LIMA; MAIA, 2014; SILVA; FARIA, 2014).

Em anuência com o Plano de Ação Político Municipal e Estatuto da Instituição, a APAS expõe suas ações que serão desenvolvidas junto ao Poder Público Municipal para assegurar os direitos plenos dos Surdos e dos Deficientes Auditivos, efetivando assim todas as suas propostas, como também articulações com órgãos particulares, empresariais, entidades não governamentais e a sociedade como um todo no sentido de cumprir com a missão de ser articuladora e defensora dessas pessoas (NOGUEIRA, 2016; APAS, 2017).

O objetivo desta proposta foi avaliar o conhecimento dos profissionais da área da saúde sobre a Linguagem Brasileira de Sinais (LIBRAS) e oferecer a esses profissionais conhecimentos essenciais para 0 atendimento de urgência $e$ emergência à pacientes com deficiência auditiva do Município de Parnaíba, Estado do Piauí. E também priorizar uma relação mais estreita e sensível entre a 
Universidade, profissionais da saúde e a população de deficientes auditivos do Município de Parnaíba, Estado do Piauí.

\section{MATERIAL E METODOS}

Alunos de graduação do curso de biomedicina e medicina, professores do curso de medicina da Universidade Federal do Piauí e membros da sociedade da cidade Parnaíba, envolvidos com ensino na área de LIBRAS, realizaram o I workshop em LIBRAS vinculado a um projeto de Extensão, para o atendimento em Urgência e emergência, na cidade de Parnaiba, Piauí, nos dias 17 e 18 de setembro de 2015, no auditório central da Universidade Federal do Piauí, Campus Ministro Reis Velloso.

Durante este evento foi aplicado, de maneira anônima, um questionário para 67 participantes, 53 antes e 14 depois das apresentações das palestras, com 11 perguntas objetivas, cada questionário, seguidos de palestras para averiguar o conhecimento da população, alunos e profissionais da área da saúde sobre a linguagem de sinais antes e após as quatro palestras, que tiveram como objetivo divulgar o conhecimento sobre sinais úteis em atendimentos de urgência e emergência médica .

Para tanto foi utilizada uma metodologia qualitativa, com característica principal de aproximação ou o vínculo com o objeto a ser pesquisado e explanado, ocorrendo sempre à definição dos fenômenos que são observados em situações reais. Sendo assim, a abordagem de pesquisa qualitativa, segundo Ludke e André (1986), é o tipo de pesquisa que respeita de forma discreta a liberdade do pesquisando, permitindo envolvimento ou inserção pessoal, intelectual ou social.

Para o desenvolvimento desse projeto, foi estruturado em três momentos, sendo o primeiro destinado à aplicação de um questionário de sondagem e avalição dos conhecimentos à priori, antes das informações dadas nas palestras. Este questionário continha 11 questões sobre sinais (em LIBRAS) e as palavras correspondentes a esses sinais (em português), para que fossem "ligadas" ou relacionadas com seu significado.

O questionário foi aplicado com o intuito de averiguar o conhecimento dos participantes da área da saúde presentes no I workshop de LIBRAS para o atendimento de urgência e emergência, sobre conceitos básicos voltados para 0 atendimento de urgência e emergência. Participaram desta pesquisa 53 profissionais da área da saúde. O critério de inclusão na pesquisa foi: o entrevistado ser profissional da área saúde. Estes participantes foram submetidos ao questionário de sondagem onde correlacionaram algumas figuras (sinais) aos seguintes conceitos: cabeça, braços, barriga, mão, boca e perna e também os sinais em LIBRAS com as palavras: diarreia, febre, vômito, tosse e gripe.

O segundo momento foi logo após a aplicação do questionário de sondagem, os participantes do evento assistiram às palestras com orientações e esclarecimentos sobre os principais sinais que podem auxiliá-los em diferentes situações, participaram de brincadeiras e assistiram filmes correlatos, também participaram de mesas redondas sobre o assunto, com a oportunidade de sanar suas dúvidas diante de profissionais a respeito do tema.

O terceiro momento foi composto da aplicação do mesmo questionário de sondagem para avaliar o que os participantes capturaram e conseguiram aprender, durante as palestras e se de alguma forma estas contribuíram para um futuro auxilio 
por parte destes profissionais aos deficientes auditivos. Este trabalho foi devidamente cadastrado e certificado pela Câmera da Universidade Federal do Piauí e protocolado sob o número, 23111.006946/2015-12.

Os dados, ou questionários foram submetidos à contagem direta, para obtenção das frequências e do número de acertos e erros antes e após a apresentação das palestras no referido evento. A metodologia estatística utilizada foi a de análise qualitativa direta, com a utilização do teste de Qui-quadrado a $5 \%$ de significância, mediante a PROC NPARWAY que foi realizada por meio do programa (SAS, 2003).

\section{RESULTADOS E DISCUSSÃO}

O primeiro momento da pesquisa, destinado a avaliação do conhecimento dos profissionais da saúde da cidade de Parnaíba, quanto a LIBRAS, através da aplicação de um exercício de sondagem (aplicação de um questionário), evidenciou o desconhecimento dos participantes da área da saúde sobre a linguagem de sinais, que se referiram a LIBRAS como mímica e/ou gestos sem muito valor para a comunicação.

A aplicação deste instrumento individual buscou identificar as dificuldades encontradas pelos profissionais da área da saúde durante o atendimento aos pacientes surdos. Do total de 53 participantes desta pesquisa inicial, 31 (58,4\%) responderam todos os testes corretamente e 22 pessoas $(41,5 \%)$ não responderam todas as questões corretamente, demonstrando ainda um desconhecimento desta linguagem (Tabela 1).

TABELA 1 - Número total, média, número de acertos e erros, porcentagens e significância de respostas de profissionais da área da saúde no I workshop de LIBRAS para o atendimento de urgência e emergência, respondidos antes e após as palestras.

\begin{tabular}{lccccccc}
\hline $\begin{array}{l}\text { Número de } \\
\text { Profissionai } \\
\text { s }\end{array}$ & Médias & Acertos & Erros & $\%$ Acerto & \%Erro & -Value P \\
\hline Antes & 53 & $7,57^{b^{*}}$ & 31 & 22 & $58,5 \%$ & $41,5 \%$ & $<.0001$ \\
Depois 14 & $9,82^{\mathrm{a}}$ & 12 & 2 & $85,8 \%$ & $14,2 \%$ & \\
\hline
\end{tabular}

Fonte: Autores, (2017).

*Médias seguidas de letras diferentes diferem significativamente pelo teste de quiquadrado a $5 \%$ de significância.

A aplicação do mesmo questionário de sondagem para avaliar o que os participantes aprenderam durante as palestras, foi observado que de 14 profissionais da área da saúde que responderam novamente o mesmo questionário, $12(85,7 \%)$ conseguiram responder todas as questões sem nenhum erro.

Aos participantes do I workshop de LIBRAS para o atendimento de urgência e emergência, foram proporcionados conhecimentos a respeito dos deficientes auditivos, as dificuldades enfrentadas por eles e os vários tipos de sinais para uma comunicação mais eficiente em casos de primeiros socorros, sinais estes que permitam estabelecer uma comunicação adequada visando estabelecer a relação médico-paciente (NOGUEIRA et al., 2016). 
Os alunos e professores que se envolveram na organização e participação do workshop tiveram a oportunidade de adquirir os conhecimentos mínimos sobre libras, despertando nestes o desejo de ampliar seus conhecimentos sobre a linguagem, sendo assim muitos procuraram capacitar-se através de cursos profissionalizantes na área de LIBRAS. É de fundamental importância abordar questões direcionadas para formação de recursos humanos para produção de conhecimento na área de Educação Especial (GLAT; PLETSCH, 2010).

Segundo os profissionais da área da saúde, que participaram do evento, o I workshop de LIBRAS para o atendimento de urgência e emergência, foi de grande valia, pois naquele momento tiveram a oportunidade de aprender e reforçar alguns sinais que poderão auxiliá-los em um contato inicial com os pacientes surdos, demonstrando com isso estar abertos a inclusão social, além de vivenciarem, pela presença na ocasião dos surdos no evento, um pouco dos relatos da realidade e as dificuldades destes, diante de atendimentos médico-hospitalares. As pessoas com problemas de audição, às vezes não são atendidas da forma correta, com isso a necessidade dos profissionais possuírem conhecimento em LIBRAS (SANTOS, 2015; SOUZA, 2017).

Uma adequada inclusão social dentro dos serviços de saúde humanizaria os serviços prestados aos portadores de necessidades especiais (TRECOSSI; ORTIGARA, 2013). Quando se trata de uma pessoa surda é perceptível à barreira de comunicação que pode comprometer a interação do paciente e do profissional, pela ausência da comunicação oral do surdo, daí a necessidade por parte dos profissionais da saúde de buscarem o conhecimento desta Língua (TRECOSSI; ORTIGARA, 2013; OLIVEIRA et al., 2015).

Os deficientes auditivos têm dificuldades para usufruir serviços básicos, como, por exemplo, acesso a hospitais, porém uma boa comunicação entre os profissionais de saúde e o usuário, torna esse profissional diferenciado, por serem capazes de entender as necessidades desse paciente e conseguirem prestar assistência adequada a eles, minimizando o desconforto (OLIVEIRA et al., 2015).

Muitos profissionais relataram ter associado o desenho com a parte do corpo na gravura, e devido a isso conseguirem uma boa quantidade de acertos, o que não significou conhecer o sinal (Tabela 1). A comunicação com pacientes surdos continua negligenciada nos sistemas de saúde e alerta-se que saber se comunicar é uma importante ferramenta para os profissionais de saúde (CHAVEIRO et al., 2010).

O nível de conhecimentos adquiridos neste estudo pelos participantes do I workshop de LIBRAS para o atendimento de urgência e emergência proporcionou uma diferença significativa no conhecimento dos profissionais presentes e avaliados $(p<0,05)$, quando foram comparados os questionários respondidos antes e após as palestras pelo teste de qui-quadrado, a $5 \%$ de significância (Tabela 1).

Como é possível observar a quantidade de participantes do I workshop de LIBRAS para o atendimento de urgência e emergência de diferentes áreas da saúde, que erraram os diferentes sinais ao responderem os questionários antes das palestras, foi significativamente $(p<.0001)$ maior quando comparado com o número de erros após as palestras (Figura 1 e Tabela 1). 


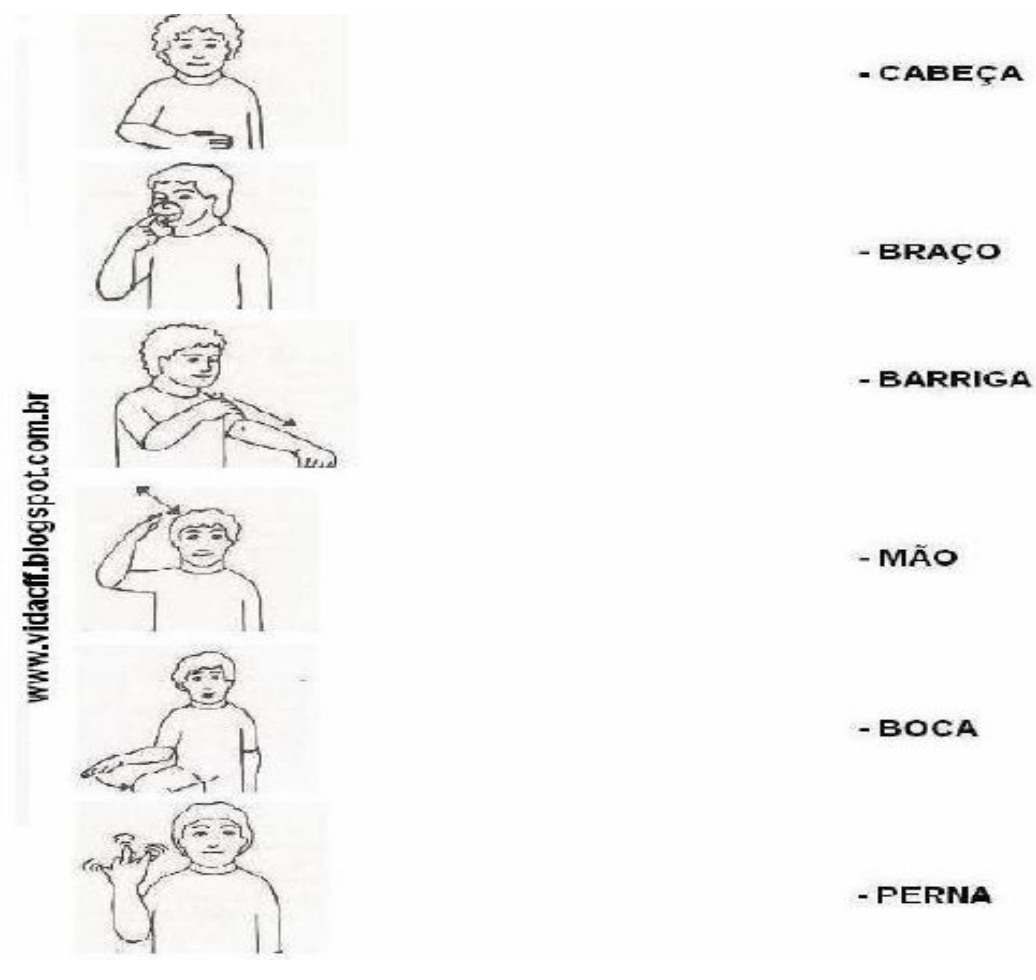

Fonte: www.vidacff.blogspot.br

FIGURA 1. Exercício de sondagem (contendo os sinais e as palavras correspondentes) aplicado aos profissionais da área da saúde antes e após o I workshop em LIBRAS para o atendimento em urgência e emergência, ocorrido na cidade de Parnaíba, Piauí no ano de 2015, na Universidade Federal do Piauí.

Dentre as 53 pessoas que responderam ao questionário, 22 pessoas erraram um ou mais sinais dentre estas: uma pessoa errou um sinal, 12 pessoas erraram dois sinais, quatro pessoas erraram três sinais, duas pessoas erraram cinco sinais e uma pessoa errou seis sinais (Tabela 2). Das 14 pessoas que responderam o questionário posteriormente às palestras somente uma errou dois sinais e uma errou três sinais, como consta na Tabela 2.

TABELA 2 - Número de profissionais da área da saúde e quantidade de sinais respondidos incorretamente antes e após as palestras.

\begin{tabular}{llll}
\hline $\begin{array}{l}\text { Número } \\
\text { profissionais }\end{array}$ & $\begin{array}{l}\text { de } \\
\text { Número de erros } \\
\text { antes } \\
\text { palestras }\end{array}$ & $\begin{array}{l}\text { Número de } \\
\text { profissionais }\end{array}$ & $\begin{array}{l}\text { Número erros após } \\
\text { as palestras }\end{array}$ \\
\hline 1 & 1 & 1 & 2 \\
12 & 2 & 1 & 3 \\
4 & 3 & - & - \\
2 & 4 & - & - \\
2 & 5 & - & - \\
1 & 6 & - & - \\
\hline Total 22 & 21 & 2 & 5 \\
\hline
\end{tabular}

Fonte: Autores (2017) 
A quantidade de alternativas respondidas incorretamente antes das palestras foi bem maior que a quantidade de alternativas incorretas respondidas após as apresentações das palestras. Isso quer dizer que as pessoas entenderam as palestras e aprenderam as configurações de mãos e sinais correspondentes às perguntas (Tabela 2).

É possível preparar os profissionais de saúde com aprendizados básicos que possam auxiliá-los no futuro, melhorando o relacionamento profissional/paciente e dessa forma contribuir para a inclusão dos deficientes auditivos (TRECOSSI; ORTIGARA, 2013).

Os sinais que obtiveram mais respostas erradas por estes 22 profissionais participantes da pesquisa antes das palestras foram sinais das palavras: vômito e tosse com 18 respostas incorretas, cada uma e o sinal para gripe, com 13 respostas erradas, diarreia (4 respostas erradas), febre (4), braço (3) e boca (1) (Tabela 3).

TABELA 3 - Sinais contidos nos questionários aplicados aos profissionais da área da saúde antes e após as palestras do I workshop de LIBRAS para o atendimento de urgência e emergência.

\begin{tabular}{|c|c|c|c|}
\hline Sinais & Significado do sinal & $\begin{array}{c}\text { Erros antes das } \\
\text { palestras }\end{array}$ & $\begin{array}{r}\text { Erros após as } \\
\text { palestras }\end{array}$ \\
\hline & VÔMITO & 18 & 2 \\
\hline & TOSSE & 18 & 2 \\
\hline & GRIPE & 13 & 1 \\
\hline \multirow{4}{*}{ 里 } & DIARRÉIA & 4 & 0 \\
\hline & FEBRE & 4 & 0 \\
\hline & BRAÇO & 3 & 0 \\
\hline & BOCA & 1 & 0 \\
\hline TOTAL & & Erros $=61$ & Erros $=4$ \\
\hline
\end{tabular}

Fonte: www.vidacff.blogspot.br e Autores, (2017) 
Após as palestras os erros foram reduzidos, pois apenas quatro profissionais erraram dois sinais, sendo dois erros para o sinal de vômito e dois para o sinal de tosse (Tabela 3). Pode-se observar na Figura 1 um total de 61 erros dos profissionais da área da saúde antes das apresentações das palestras, frente a um total de quatro sinais errados após as palestras, o que evidencia a diferença de conhecimento antes e após as palestras serem ministradas.

Um trabalho realizado durante as atividades da IV Semana Acadêmica de Medicina, promovida pelos alunos do Curso de Medicina da Universidade Federal de Tocantins, corrobora com este trabalho, estes constaram que o pré-teste aplicado no minicurso, evidenciou o desconhecimento dos alunos sobre a cultura surda e a linguagem de sinais, e no pós-teste, a mudança foi significativa (LEVINO et al., 2013).

Estatisticamente o nível de conhecimento dos profissionais da área da saúde neste estudo, foi significativamente mais alto após as palestras, sendo visível que muitos estão tentando se aproximar dos surdos, e quando tentam, conseguem identificar os gestos, por isso, existe a necessidade de produzir um material prático contendo os sinais mais utilizados na área da saúde, pois eles acreditam que este material auxiliaria e facilitaria na qualificação dos diversos profissionais desta área, e seria uma grande conquista para difusão da linguagem de sinais (CARVALHO FILHA et al., 2015).

\section{CONCLUSÃO}

Os profissionais da área da saúde do Município de Parnaíba, Estado do Piauí não estão preparados para receber os pacientes auditivos por não dominarem a Linguagem Brasileira de Sinais (LIBRAS), por isso não oferecem atendimentos adequados a esses pacientes, que muitas vezes precisam pagar por interpretes para ser assegurado um bom atendimento. Este Workshop ofereceu a esses profissionais conhecimentos básicos e essenciais para o atendimento de urgência e emergência e atentou os membros da sociedade pertencentes à área da saúde sobre a importância dos conhecimentos básicos para um bom atendimento a pacientes com deficiência auditiva.

\section{REFERÊNCIAS}

APAS - Associação de Pais e Amigos dos Surdos de Parnaíba, Piauí - Sobre a Instituição. Parnaíba, 2016. Acesso em 07/11/2017. Disponível em: http://www.apascresp.com/blank-6.

BERNARDES, L. C. G; MAIOR. I, M, M, L; SPEZIA. C, H; ARAUJO. T, C, C, F. Pessoas com Deficiência e Políticas de saúde no Brasil: Reflexões Bioéticas. Ciência e Saúde Coletiva, v. 14, n. 1, p. 31-38, 2009. http://www.scielo.br/pdf/csc/v14n1/a08v14n1.pdf.

CARVALHO FILHA, F. S. S.; SILVA, S. R.; LANDO, G. A. Cuidado ao surdo: conexões com o direito à saúde. Revista Ciência \& Saberes, v. 1, n. 1, p. 31-38, 2015. https://idonline.emnuvens.com.br/id/article/view/589.

CHAVEIRO, N; BARBOSA, M.A; PORTO. C, C; MUNARI.D, B; MEDEIROS. M. et al. Atendimento à pessoa surda que utiliza a língua de sinais, na perspectiva do 
profissional da saúde. Cogitare Enfermagem, v. 15, n.4, p. 639-645, 2010.

https://revistas.ufpr.br/cogitare/article/download/20359/13520.

CRUZ. R, C, V; SOUZA. V, A VILELA. F, M, A. O tradutor e intérprete de língua brasileira de sinais/língua portuguesa (TILSP) - Boletim Técnico IFTM, UberabaMG, ano 2, n.3, p.06-09, 2016. http://editora.iftm.edu.br/index.php/boletimiftm/article/view/217/92.

GLAT, R.; PLETSCH, M. D. O papel da Universidade no contexto da política de Educação Inclusiva: reflexões sobre a formação de recursos humanos e a produção de conhecimento. Revista Brasileira de Educação Especial, v.23, n. 38, p. 345356, 2010. http://www.ufsm.br/revistaeducacaoespecial.

LEVINO. D, A; SOUZA. E, B; CARDOSO. P, C; SILVA.A, C CARVALHO. A, E, T, M. Libras na Graduação Médica: o Despertar para uma Nova Língua. Revista Brasileira de Educação Médica, v.37, n.2, p. 291 - 297, 2013. http://www.scielo.br/pdf/rbem/v37n2/18.pdf.

LIMA, J. R.; MAIA, C. S. A. Importância do ensino de libras para os profissionais da saúde. Comunicação Saúde e Educação, v.13, n. 28, p. 177-88, 2014. https://www.editorarealize.com.br/revistas/conacis/trabalhos/Modalidade_2datahora 24_03_2014_16_07_12_idinscrito_249_290cfa756b2b36b2792260885e0fcd26.pdf.

LUDKE, M.; ANDRÉ, M. E. D. A. Pesquisa em Educação: abordagens qualitativas. São $\quad$ Paulo: 1986. https://periodicos.pucpr.br/index.php/dialogoeducacional/article/viewFile/3886/3799.

MOREIRA, P. A. L. O fator linguístico na aprendizagem e desenvolvimento cognitivo da criança surda. Revista Virtual de Cultura Surda e Diversidade, 2017. Disponível em: <http://www.editora-arara-azul.com.br/revista/03/compar1.2.php> Acesso em 08 out. 2017.

NOGUEIRA. G, C; SCHOELLER. S, D; SOUZA. F, R, S; PADILHA. M, I; BREHME. L, C, F. et al, Perfil das pessoas com deficiência física e Políticas Públicas: a distância entre intenções e gestos. Ciência \& Saúde Coletiva, 21(10):3131-3142, 2016. DOI: $10.1590 / 1413-812320152110.17622016$.

OLIVEIRA, Y. C. A. O.; CELINO, S. D. M. C.; COSTA, G. M.C. Comunicação como ferramenta essencial para assistência à saúde dos surdos. Physis: Revista de $\begin{array}{llllll}\text { Saúde } & \text { Coletiva, } & \text { v. } & 25, & \text { n.1, } & \text { p. }\end{array}$ http://www.scielo.br/pdf/physis/v25n1/0103-7331-physis-25-01-00307.pdf.

ONU - Organização das Nações Unidas. Pessoas com deficiência. Nações Unidas no Brasil, 2017. Acesso em 07/11/2017. Disponível em: https://nacoesunidas.org/acao/pessoas-com-deficiencia/.

QUEIROZ, M. Panorama da interpretação em contextos médicos no Brasil: Perspectivas. Tr a d Te r m , São Paulo, v. 23, Setembro. 2014, p. 193-223. DOI: https://doi.org/10.11606/issn.2317-9511.tradterm.2014.85577. 
SANTOS. P, R, A. Percepção da equipe de saúde, discentes e usuários sobre a comunicação com indivíduos surdos na atenção primária. 2015. $51 \mathrm{f}$. Dissertação (Mestrado) - Universidade Federal do Rio Grande do Norte, Natal, RN, 2015. https://repositorio.ufrn.br/jspui/handle/123456789/20208.

SAS - Statistical Analysis System. Sas Institute. User's Guide Cary, NC: SAS Institute Inc. 2003.

SILVA, F. F.; FARIA, C. C. C. O deficiente auditivo e as dificuldades na comunicação com profissionais de saúde. Revista Perquirere, v.11, n.2, p. 190-201. 2014. http://perquirere.unipam.edu.br/documents/23456/612187/O+deficiente+auditivo+e+ as++dificuldades+na++comunica $\% \mathrm{C} 3 \% \mathrm{~A} 7 \% \mathrm{C} 3 \% \mathrm{~A} 30+\mathrm{Com}+$ profissionais+de+sa\%C 3\%BAde.pdf

SOUZA, E.M.; ALMEIDA, M.A.P.T. Atendimento ao surdo na atenção básica: perspectiva da equipe multidisciplinar. Id on Line Revista Multidisciplinar e de Psicologia, vol.10, n.33, p. 72-82. ISSN: 1981-1179. 2017. https://idonline.emnuvens.com.br/id/article/view/589/874.

TRECOSSI, M. O.; ORTIGARA, E. P. F. Importância e eficácia das consultas de enfermagem ao paciente surdo. Revista de Enfermagem, v. 9, n. 9, p. 60-69, 2013. http://revistas.fw.uri.br/index.php/revistadeenfermagem/article/view/938. 